\title{
PREVALENCIA DE CARIES EN ESCOLARES DE CHIQUINTAD CUENCA 2016
}

\section{PREVALENCE OF CARIES IN SCHOOLCHILDREN OF CHIQUINTAD CUENCA 2016}

\author{
Macao-Campoverde Juan Pablo ${ }^{1 *}$ Reinoso-Vintimilla Napoleón. $^{2}$ \\ ${ }^{1}$ Odontólogo Rural del Ministerio de Salud Pìblica del Ecuador. Ecuador \\ 2 Catedrático de la Carrera de Odontología de la Universidad Católica de Cuenca. Ecuador. \\ *jpmacao1992@gmail.com
}

\begin{abstract}
Resumen
OBJETIVO: Determinar la prevalencia de caries dental mediante el índice CPOD en niños de 12 años de edad de la parroquia Chiquintad del cantón Cuenca. MATERIALES Y MÉTODOS: la metodología aplicada fue de tipo cuantitativo, el diseño fue descriptivo y la técnica observacional, retrospectivo. La muestra estuvo constituida por 100 escolares de 12 años. Los datos se obtuvieron de la base de datos EPI INFO 7.1, el diagnóstico fue realizado por operadores que estuvieron calibrados bajo criterios de CPOD. RESULTADOS: el total de enfermos con lesión cariosa fue del $77 \%$ y el $23 \%$ se encontraron sanos. La prevalencia de lesión cariosa de acuerdo al sexo fue mayor en el sexo masculino con un $81 \%$, mientras tanto que en el sexo femenino fue del $74 \%$. Las piezas dentales que presentaron caries dental fueron 160 en el sexo femenino que equivalen al $76 \%$ y en el sexo masculino fueron 147 piezas dentales que presentarón caries dental, lo que equivale a un $74 \%$; dientes perdidos en el sexo femenino fueron 4 piezas dentales, lo que equivale al $2 \%$ y en el sexo masculino fue 1 pieza dental, lo que equivale al $1 \%$, dientes obturados fueron 46 en el sexo femenino, lo que equivale al $22 \%$ y 51 piezas dentales obturadas que equivalen al $26 \%$ en el sexo masculino. CONCLUSIÓN: Los escolares de 12 años de edad del sexo masculino presentaron una mayor prevalencia de lesión cariosa en relación al sexo femenino.
\end{abstract}

Palabras clave: Lesión cariosa, prevalencia, epidemiología.

\begin{abstract}
OBJECTIVE: To determine the prevalence of dental caries using the CPOD index in children aged 12 years of age in the Chiquintad parish of Cuenca. MATERIALS AND METHODS: the applied methodology was of quantitative type, the design was descriptive and the observational technique, retrospective. The sample consisted of 100 schoolchildren aged 12 years. The data were obtained from the EPI info 7.1 database, the diagnosis was made by operators that were calibrated according to CPOD criteria. RESULTS: the total number of patients with carious lesion was $77 \%$ and $23 \%$ were healthy. The prevalence of carious lesion according to sex was greater in the male sex with $81 \%$, while in the female sex it was $74 \%$. The dental pieces that presented dental caries were 160 in the female sex that is equivalent to $76 \%$ and in the male sex were 147 dental pieces that present dental caries, which is equivalent to $74 \%$; Teeth lost in the female sex were 4 and dental pieces, equates to $2 \%$ and in the male sex was 1 tooth, which is equivalent to $1 \%$, teeth sealed were 46 in the female sex, which equals $22 \%$ and 51 teeth sealed, equivalent to $26 \%$ in males. CONCLUSION: Schoolchildren of 12 years of age male present a higher prevalence of carious lesion in relation to female sex.
\end{abstract}

Key words: carious lesion, prevalence, epidemiology.

\section{INTRODUCCIÓN}

Según la OMS (Organización Mundial de la Salud) estableció a la lesión cariosa como una alteración localizada de origen multifactorial que inicia su desarrollo degenerativo con la destrucción y desmineralización de la estructura dura del diente y que llega a progresar desarrollándose una cavitación en el mismo", ${ }^{1-3}$ por lo tanto se determina que la lesión cariosa ocasiona varias alteraciones en el sistema estomatognático como son: maloclusiones, alteraciones en la deglución, fonación, estética y trastornos de la articulación si no es atendido tempranamente, por lo que afecta tanto en la salud bucal como en la vida en general de personas de distintas edades. La presente investigación se orienta en la temática de estudio sobre la prevalencia de caries dental según el índice CPOD en niños de 12 años de edad de la parroquia "Chiquintad" del cantón Cuenca de la provincia 
del Azuay. Se ha determinado que la lesión cariosa es una evolución que implica una inestabilidad entre la superficie y subsuperficie de la pieza dental y como también del biofilm microbiano. Esta inestabilidad se hace evidente cuando se genera una desmineralización de las estructuras que forman parte de una pieza dental, que si no es controlado y detectado a tiempo, provoca le pérdida de la estructura de la superficie del esmalte, y a su vez en mayor grado de severidad también logra destruir a la dentina y por consiguiente a la pulpa, finalizando con la pérdida de los tejidos duros de la pieza dental. ${ }^{4-10}$

\section{MATERIALES Y MÉTODOS}

Se realizó un estudio descriptivo, transversal de análisis cuantitativo. La totalidad de la muestra correspondió a 100 niños que tenían 12 años de edad o que cumplieron hasta diciembre 31 del 2016 y aquellos que presentaron el consentimiento informado de los padres y el asentimiento de los niños pertenecientes a las escuelas de Chiquintad de la ciudad de Cuenca; para la recolección de datos se empleó los siguientes criterios de inclusión: estudiante de ambos sexos que se encontraban matriculados en los centros de educación primaria de la parroquia Chiquintad y aquellos que estaban dentro de la edad establecida.

El estudio se basó en la calibración mediante el índice CPOD, el cual es utilizado para proporcionar información sobre el número de piezas dentales que presentan lesión cariosa que existe en la población infantil, con el fin de implementar métodos para la prevención de la salud bucodental. Los examinadores fueron estudiantes de quinto año de la Carrera de Odontología de la Universidad Católica de Cuenca los cuales estuvieron bajo la tutoría de un epidemiólogo especializado en el tema. El examen clínico de los calibradores se basó en el cuadro de criterios de clasificación de hallazgos clínicos de caries.(Tabla 1$)^{11-15}$

Al finalizar el examen bucal de los escolares se evidenció un alto nivel de incidencia cariosa por lo que se puso a conocimiento de cada uno de los evaluados su hallazgo clínico y a su vez se les informó que es necesario implementar este tipo de medidas de prevención de manera que sean detectadas a tiempo y se logre un tratamiento adecuado y menos invasivo.

\section{RESULTADOS.}

El $53 \%$ perteneció al sexo femenino y un $47 \%$ al sexo masculino en escolares de 12 años en la parroquia Chiquintad.

Existió una mayor prevalencia de caries dental con el $77 \%$ y solo el $23 \%$ se encontraron sanos en los escolares de 12 años en la parroquia Chiquintad.

En el sexo femenino existió el $76 \%$ de piezas dentales con lesión cariosa, el $2 \%$ de dientes perdidos y el $22 \%$ de piezas dentales obturadas, y en el sexo masculino, se encontró
Tabla 1. Cuadro de criterios de clasificación de hallazgos clínicos.

\begin{tabular}{ll}
\hline Código & Condición dental \\
\hline 0 & Sano \\
1 & Cariado \\
2 & Obturado y con caries \\
3 & Obturado ok \\
4 & Pérdida por caries \\
5 & Pérdida por otros motivos \\
6 & Sellantes presentes \\
7 & Corona o pilar de puente \\
8 & Diente permanente no erupcionado \\
9 & No registrable \\
\hline
\end{tabular}

Grf. 1. Distribución de escolares según el sexo de la población escolar de 12 años de la parroquia Chiquintad.

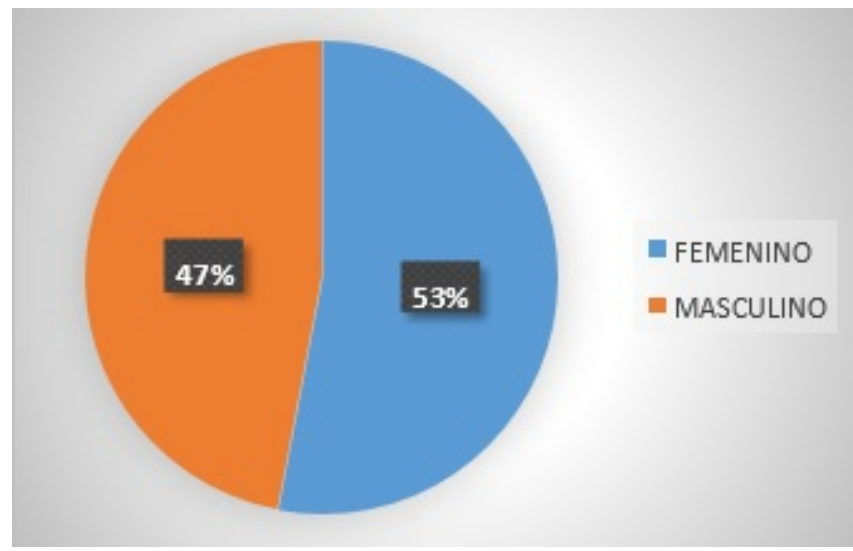

el $74 \%$ de dientes con caries dental, el $1 \%$ de piezas dentales perdidas y el $26 \%$ de piezas dentales obturadas, en escolares de 12 años en la parroquia Chiquintad.

El sexo femenino arrojó los siguientes porcentajes $10 \%$ bajo riesgo, $20 \%$ mediano riesgo y el $70 \%$ alto riesgo, mientras tanto que en el sexo masculino $6 \%$ bajo riesgo, $19 \%$ mediano riesgo y el $54 \%$ alto riesgo, siendo el nivel de severidad "alto riesgo" el más predominante en ambos géneros en los escolares de 12 años en la parroquia Chiquintad.

La prevalencia de caries dental de acuerdo al sexo se encontró con mayor prevalencia en el sexo masculino con un porcentaje del $81 \%$ con relación al sexo femenino que fue del $74 \%$, en escolares de 12 años en la parroquia Chiquintad. 
Grf. 2. Prevalencia de caries dental de la población escolar de 12 años de la parroquia Chiquintad.

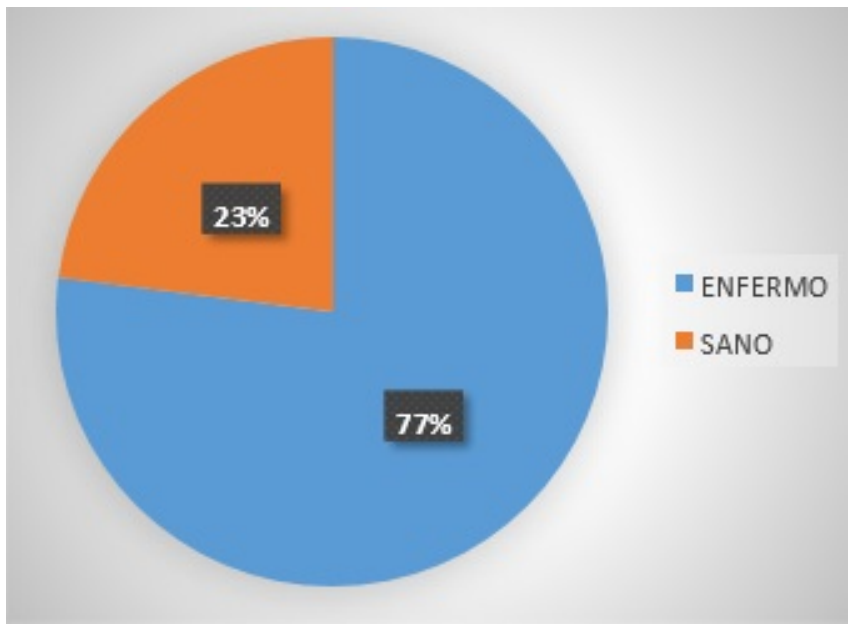

Grf. 3. Frecuencia de dientes cariados, perdidos y obturados según el índice CPOD en los escolares de 12 años de edad de Chiquintad.

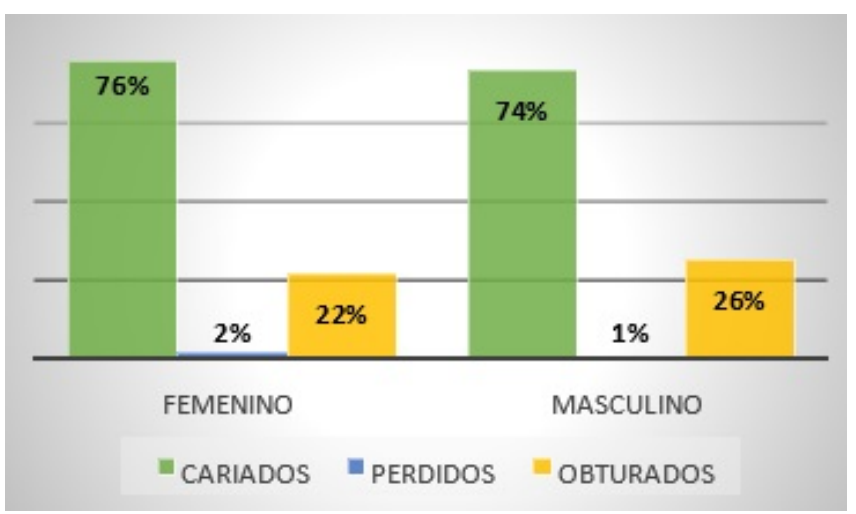

\section{DISCUSIÓN.}

De acuerdo a Villalobos, ${ }^{16-20}$ que realizó un estudio en México en Navolato, Sinaloa en escolares de 6 a 12 años, donde evidenció un porcentaje de $3.7 \%$ libre de caries para la dentición en niños de 6 años y en igual porcentaje en niños de 12 años. En cuanto a la prevalencia de caries se manifestó en mayor porcentaje siendo un $90 \%$ en los 6 años de edad y el $82 \%$ en los 12 años de edad. Existe una gran similitud con el estudio realizado en Ecuador en la parroquia de Chiquintad del cantón Cuenca en el 2016, debido a que el porcentaje que reveló en la prevalencia de caries fue del $77 \%$ y una gran diferencia en el porcentaje de libre de caries que equivale al $23 \%$, en escolares de 12 años. En Venezuela, Morelia, ${ }^{21,22}$ en el 2014 realizó un estudio descriptivo transversal, donde estudió a 445 niños desde 5 hasta 14 años de edad en donde el $77,63 \%$ presenta lesión cariosa del total de la muestra
Grf. 4. Niveles de severidad de CPOD según el género de la población escolar de 12 años de la parroquia Chiquintad.

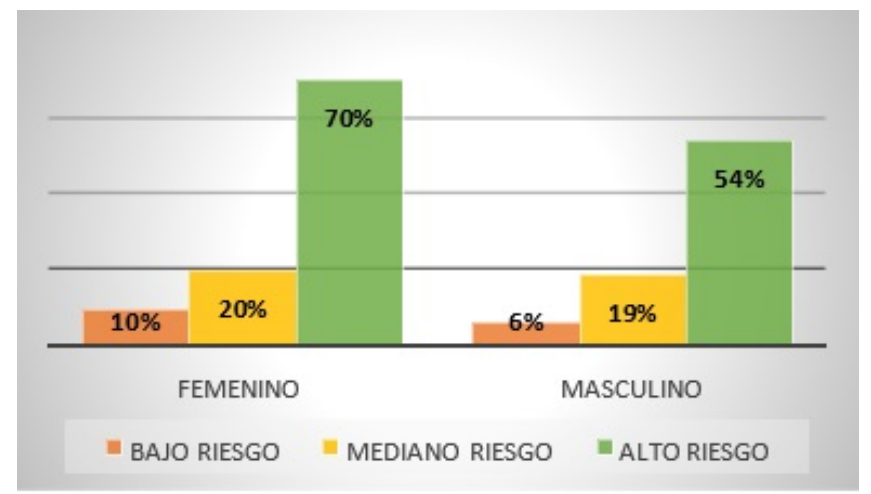

Grf. 5. Prevalencia de caries dental en base al índice CPOD según el sexo en los escolares de 12 años de edad de la parroquia Chiquintad.

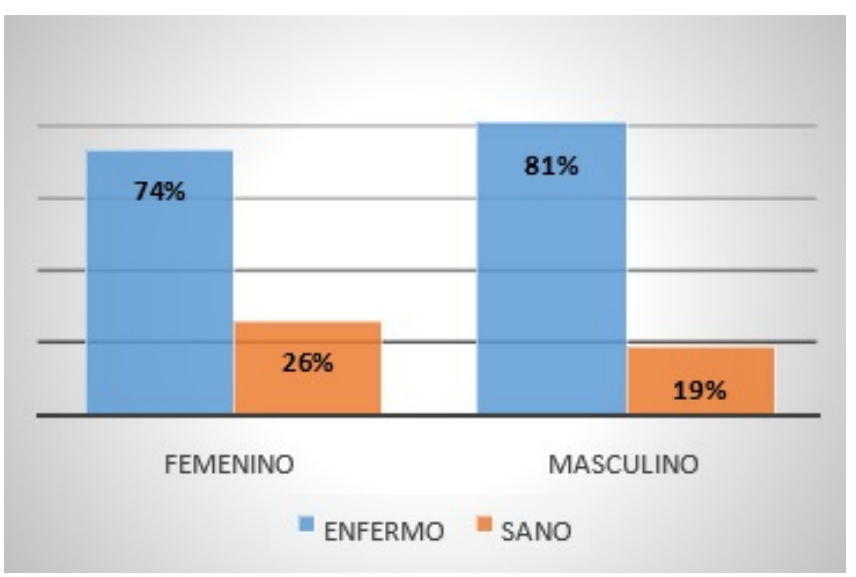

estudiada; el 53,91\% de prevalencia en dentición primaria y el $51,45 \%$ en permanente. El índice CPOD reveló un promedio de 1,4 que equivale a un nivel de "bajo riesgo". A comparación con los resultados emitidos por el estudio epidemiológico realizado en el año 2016, en la parroquia Chiquintad del cantón Cuenca, reveló que existe un nivel de severidad de "alto riesgo" en el índice CPOD, en el sexo femenino con el $70 \%$ y en el sexo masculino con el $54 \%$. El estudio que realizó González, ${ }^{23,24}$ en el año 2011 en Santiago de Chile, en donde reveló que los infantes presentaron un mayor porcentaje de dientes cariados con relación a los otros componentes de CPOD, en donde existió una prevalencia del $63,9 \%$ de caries dental indistintamente del sexo y ubicación geográfica de residencia. De igual manera manifestó el estudio realizado en Ecuador en el año 2016 en la parroquia Chiquintad, donde también existió una mayor prevalencia de dientes cariados con relación a los otros componentes del índice de CPOD, a diferencia que en el estudio realizado en 
Ecuador se dividió la prevalencia según el sexo, siendo el 12 Ministerio de Salud de la Nación. Indicadores Epidemioló$76 \%$ en el sexo femenino y el $74 \%$ en el sexo masculino, en niños de 12 años.

\section{CONCLUSIÓN.}

El estudio realizado en los escolares de 12 años de la 14 parroquia Chiquintad reveló que la caries dental presenta un nivel de severidad de "alto riesgo", siendo mayor en el grupo poblacional masculino. Los resultados de la investigación evidencian claramente la necesidad de tratamientos tanto preventivos como restaurativos de la población evaluada.

Conflicto de intereses y financiamiento Los autores declaran no tener conflicto de intereses, haber cumplido con los requisitos de autoría y haber autofinanciado este artículo.

\section{Referencias}

1 Palomer L. Caries dental en el niño. Una enfermedad contagiosa. Revista chilena de pediatría. 2006; 77(1).

2 Villalobos J, Medina C, Vallejos A, Espinoza J. Caries dental en escolares de 6 a 12 años de Navolato, Sinaloa: resultados preliminares. Biomed. 2005;(217-219).

3 Díaz S, González F. Prevalencia de caries dental y factores familiares en niños escolares de Cartagena de Indias, Colombia. Salud pública. 2010

4 Márquez M, Rodríguez R, Rodríguez Y, Estrada G, Aroche A. Epidemiología de la caries dental en niños de 6-12 años en la Clínica Odontológica "La Democracia". MEDISAN. 2009; 13(5).

5 Paiva S, Álvarez E, Abanto J, Cabrera A, López R, Masoli $\mathrm{C}$, et al. Relatorios de la mesa de representantes de sociedades de Odontopediatría de los países Latinoamericanos. Revista de odontopediatria latinoamericana. 2014.

6 Portilla J, Pinzón M, Huerta E, Obregón A. Conceptos actuales e investigaciones futuras en el tratamiento de la caries dental y control de la placa bacteriana. Revista Odontológica Mexicana. 2010; 14(4).

7 Núñez D, García L. Bioquímica de la caries dental. Revista Habanera de Ciencias Médicas. 2010; 9(2).

8 Hernández A, Espeso N, Reyes F, Rodríguez L. Intervención educativo-curativa para la prevención de caries dental en niños de cinco a 12 años. Revista Archivo Médico de Camagüey. 2010 Noviembre - Dicembre; 14(6).

9 Flores M, Montenegro B. Relación entre la frecuencia diaria de consumo de azúcares extrínsecos y la prevalencia de caries dental. Rev Estomatológica Herediana. 2014; 15(1).

10 Olmos P, Piovesan S, Musto M, Lorenzo S, Álvarez R, Massa F. Caries dental. La enfermedadoral más prevalente: Primer Estudio poblacional en jóvenes y adultos uruguayos del interior del país. Odontoestomatologica. 2013; 15.

11 Gutiérrez P, García A, Santacoloma S, Mejía J. Caries dental: ¿influyen la genética y la epigenética en su etiología? Universitas Odontologica. 2013 Julio- Diciembre; 32(69).

13 Da Silva F, Massao J, Da Gama S. La prevalencia de caries dental y evaluación de factores de riesgo en niños que participaron o no. Acta odontol. venez. 2012; 50(4).

14 Piovano S, Squassi A, Bordoni N. Estado del arte de indicadores para la medición de caries dental. Revista de la Facultad de Odontología (UBA). 2010; 25(58).

15 Morelia A, Simancas Y, Salas M, Díaz N, Rome Y. Prevalencia y experiencia de caries en niños en edad escolar. Acta Bioclínica. 2014; 4(7).

16 Alarcón N, Aguilera C. Relación entre prevalencia de caries dental, índice de higiene oral y estado nutricional en niños preescolares de 3 a 5 años 11 meses del sector Pedro del Río , Concepción ,VIII Región, Chile 2014.

17 Narváez H , López E. Determinantes sociales y su relación con caries en niños de 1 a 5 años de la fundación "niños de maría" de la ciudad de quito durante el año lectivo 20112012. Odontología. 2015; 17(1).

18 González C, Franz L, Sanzana N. Determinantes de salud oral en población de 12 años. Rev. Clin. Periodoncia Implantol. Rehabil. Oral. 2011; 4(3)

19 Alvarez L, Romá P. Prevalencia de caries en niños de 6 y 12 años de colegios asignados al Centro de Salud Familiar N4, Dra. María Latiffe S. de Rancagua. Revista Dental de Chile. 2013; 104(3).

20 Villavicencio E. Proyecto: Prevalencia de Caries en escolares de 12 años en Cuenca 2016. researchgate.net

21 Encalada L. Estudio piloto comparativo de salud oral relacionada a la calidad de vida en escolares del cantón cañar ecuador 2015. oactiva.ucacue.edu.ec. 2016 Enero; 1(1).

22 Reinoso N, Villavicencio E. Caries dental en escolares de 12 años de la parroquia Sayausí Cuenca Ecuador. oactiva.ucacue.edu.ec. 2016; 1(1).

23 Villavicencio E. [Internet]. El tamaño muestral en tesis de post grado. ¿cuantas personas debo encuestar? researchgate [Internet]. 2016 [citado 31Ags 2016]; pag 1-4.

24 Villavicencio E, et al. Pasos para la planificación de una investigación clínica. oactiva.ucacue.edu.ec. 2016; 1(1):1-5.

Recibido: 16 de enero de 2017.

Aceptado: 14 de marzo de 2017. 